\title{
Defective adipose tissue development associated with hepatomegaly in cathepsin E-deficient mice fed a high-fat diet
}

\author{
Tomoko Kadowakia, Mizuho A. Kido ${ }^{b}$, Junko Hatakeyama ${ }^{b, 1}$, Kuniaki Okamotoc, Takayuki \\ Tsukuba $^{\mathrm{c}, *}$ and Kenji Yamamoto ${ }^{\mathrm{d}}$
}

${ }^{a}$ Research Promotion Section, Nagasaki University, Nagasaki 852-8521, Japan

${ }^{b}$ Department of Molecular Cell Biology and Oral Anatomy, Graduate School of Dental Science, Kyushu University, Fukuoka 812-8582, Japan

${ }^{c}$ Division of Oral Pathopharmacology, Graduate School of Biomedical Sciences, Nagasaki University, Nagasaki 852-8588, Japan

${ }^{d}$ Proteolysis Research Laboratory, Graduate School of Pharmaceutical Sciences, Kyushu University, Fukuoka 812-8582, Japan

Running title: Fat-induced cathepsin E-deficient mice

Key words: cathepsin E, high-fat diet, adipose tissue

${ }^{1}$ Present address: Department of Odontology, Section of Operative Dentistry and Endodontology, Fukuoka Dental College, Sawara-ku, Fukuoka 814-0193, Japan

*Corresponding author: Dr. Takayuki Tsukuba

Division of Oral Pathopharmacology, Graduate School of Biomedical Sciences, Nagasaki University, 1-7-1 Sakamoto, Nagasaki 852-8588, Japan

Tel: 81-95-819-7652; Fax: 81-95-819-7655; E-mail: tsuta@nagasaki-u.ac.jp 


\begin{abstract}
Cathepsin $\mathrm{E}$ is an intracellular aspartic proteinase, which is predominantly distributed in immune-related and epithelial cells. However, the role of the enzyme in adipose tissues remains unknown. In this study, we investigated the characteristics of cathepsin E-deficient (Cat ${ }^{-/}$) mice fed a high-fat diet (HFD), as a mouse model of obesity. HFD-fed CatE $E^{-/-}$mice displayed reduced body weight gain and defective development of white adipose tissue (WAT) and brown adipose tissue (BAT), compared with HFD-fed wild-type mice. Moreover, fat-induced CatE$^{-/-}$ mice showed abnormal lipid accumulation in non-adipose tissues characterized by hepatomegaly, which is probably due to defective adipose tissue development. Detailed pathological and biochemical analyses showed that hepatomegaly was accompanied by hepatic steatosis and hypercholesterolemia in HFD-induced $\mathrm{CatE}^{-/-}$mice. In fat-induced $\mathrm{CatE}^{-/-}$mice, the number of macrophages infiltrating into WAT was significantly lower than in fat-induced wild-type mice. Thus, the impaired adipose tissue development in HFD-induced $\mathrm{CatE}^{-/}$mice was probably due to reduced infiltration of macrophages and may lead to hepatomegaly accompanied by hepatic steatosis and hypercholesterolemia.
\end{abstract}

\title{
Abbreviations
}

$\mathrm{CatE}^{-{ }^{-}}$, cathepsin E-deficient; ND, normal diet; HFD, high-fat diet; CHO, cholesterol; LDL, low density lipoprotein; SAA, serum amyloid A; white adipose tissue, WAT; brown adipose tissue, BAT 


\section{Introduction}

Cathepsin E (EC 3.4.23.34) is an intracellular aspartic proteinase that is mainly localized within cell endosomes or lysosomes [1,2]. Cathepsin E is predominantly found in immune-related cells, including macrophages, dendritic cells, and microglia $[3,4]$ or in the epithelial cells of the gastrointestinal mucosa and skin [5,6]. To investigate the physiological or pathological roles of the proteinase in vivo, we have previously generated cathepsin E-deficient $\left(\mathrm{CatE}^{-/-}\right)$mice. CatE ${ }^{-/-}$mice spontaneously developed atopic dermatitis-like skin lesions when kept under conventional conditions, but not under specific pathogen-free conditions [7]. Recently, we reported that macrophages derived from $\mathrm{CatE}^{-/-}$mice display a novel type of lysosomal storage disorder, characterized by accumulation of lysosomal membrane glycoproteins such as LAMP-1 and LAMP-2, in cells with a concomitant elevated lysosomal pH [8]. In particular, CatE ${ }^{-/-}$macrophages exhibit various abnormalities, such as reduced bactericidal activity [9], trafficking defects for both membrane and soluble proteins [10], and impaired TNF- $\alpha$ or IL-6 production [9]. Although cathepsin E deficiency probably causes diseases other than skin lesions, other roles of cathepsin E in vivo remain unknown. However, during a series of experiments comparing wild-type and $\mathrm{CatE}^{-{ }^{--}}$mice, we observed a modest decrease in the body weight of $\mathrm{CatE}^{-/-}$mice compared to wild-type mice. These findings prompted us to investigate whether cathepsin E deficiency also causes a lean phenotype.

To date, several studies have reported that endosome or lysosome dysfunction leads to a deficiency in adipose deposition. For example, mice deficient in lysosomal acid lipase, which hydrolyzes triglycerides and cholesterol (CHO) esters in lysosomes, exhibit massive accumulation of triglycerides and $\mathrm{CHO}$ esters in the liver, spleen, and intestines, and lack adipose tissues, despite being born with normal fat distribution [11]. Moreover, mice with different lysosomal storage diseases (mucopolysaccharidosis (MPS) type I [MPS I], MPS IIB, MPS VII, Niemann-Pick type A/B, and infantile neuronal ceroid lipofuscinosis) commonly show 
adipose storage deficiency but have differing serum levels of leptin and insulin [12]. These findings indicate that lysosomal storage dysfunction causes deficiency in adipose deposition regardless of the specific enzymes affected, even under normal diet (ND) conditions, but not under high-fat diet (HFD) conditions.

In this study, we demonstrate that HFD-induced $\mathrm{CatE}^{-/-}$mice have less adipose tissue and display a phenotype of hepatomegaly accompanied with heptic steatosis and hypercholesterolemia.

\section{Materials and Methods}

\subsection{Mice}

Wild-type and Cat ${ }^{-/}$mice with the C57BL/6 genetic background were used as described previously [7,8]. Animals were maintained under specific pathogen-free conditions according to the guidelines of the Japanese Pharmacological Society. All animals and experiments were approved for use by the Animal and Microbiological Research Committees of the Graduate School of Dental Science, Kyushu University, or the Graduate School of Biomedical Sciences, Nagasaki University. All experiments were performed with age-matched male CatE $E^{-/-}$mice and wild-type littermates. Eight-week-old male mice were fed for 16 weeks with regular chow (ND) or an HFD containing 25\% milk casein, $14 \%$ beef fat and $14 \%$ lard.

\subsection{Histopathological analysis}

Mice were anesthetized with ether, and blood was drawn for serum analysis. All organs were fixed with $10 \%$ formalin and processed for pathological analysis. The fixed organs were sectioned and stained with hematoxylin and eosin (H\&E). Fixed aortas were dissected and prepared for either Oil Red O staining using standard techniques or for paraffin embedding and H\&E staining. 
For immunohistological analysis, adipose tissue was fixed with $4 \%$ paraformaldehyde in phosphate buffer.

\subsection{Immunohistochemistry}

Immunohistochemistry was performed as described previously [13]. Briefly, 20- $\mu \mathrm{m}$ cryosections were treated with $0.3 \%$ Triton X-100 in PBS for 30 min, followed by $0.3 \% \mathrm{H}_{2} \mathrm{O}_{2}$ in PBS for 60 min. After treatment with $10 \%$ non-immune goat serum, the sections were incubated with rabbit anti-F4/80 monoclonal antibody (used at a dilution of 1:500; Serotec, Oxford, UK) overnight at $4^{\circ} \mathrm{C}$, followed by immunostaining with an avidin-biotin-peroxidase complex (ABC) kit (Vector Laboratories, Burlingame, CA, USA). The sections were incubated for 5 min with $0.02 \%$ 3,3'-diaminobenzidine tetrahydrochloride (DAB; Dojindo Laboratories, Kumamoto, Japan) and 0.006\% $\mathrm{H}_{2} \mathrm{O}_{2}$ in $0.05 \mathrm{M}$ Tris buffer (pH 7.6), followed by hematoxylin staining.

\subsection{Biochemical and lipid analyses}

The concentrations of total CHO, high-density lipid (HDL)-CHO, non-esterified fatty acid (NEFA), glucose, and insulin, were measured using an enzymatic assay kit (Cholesterol E-test, HDL Cholesterol E-test, NEFA C-Test, Glucose CII test, and Insulin-EIA test: Wako Pure Chemical Industries, Osaka, Japan). The levels of low-density lipid (LDL)-CHO were calculated based on the results of total CHO, HDL-CHO, and triglycerides. The levels of serum amyloid A (SAA) were measured by using an ELISA kit (Shibayagi, Gunma, Japan). The serum levels of thiobarbituric acid reactive substances (TBARS) were measured using an assay kit (Cayman Chemical Co, MI, USA).

\subsection{Statistical analysis}

The Student's $t$-test was used to determine the statistical significance of observed differences. 


\section{Results}

\subsection{HFD-induced $\mathrm{CatE}^{-/-}$mice showed fat tissue depletion and hepatomegaly}

We first investigated body weight gain in wild-type and CatE ${ }^{-/-}$mice fed an ND (Fig. 1A) or an HFD (Fig. 1B). The ND-fed $\mathrm{CatE}^{-/-}$mice displayed slightly lesser body weight gain than ND-fed wild-type mice, but this difference was statistically insignificant (Fig. 1 A). However, when mice were fed an HFD from weeks 8 to 28, the $\mathrm{CatE}^{-{ }^{-}}$mice exhibited significantly lesser weight gain than wild-type mice (Fig. 1B). Food intake did not differ between wild-type and $\mathrm{CatE}^{-/-}$mice maintained either on an ND or HFD (data not shown).

We then dissected the chest and abdominal parts of 24-week-old wild-type and $\mathrm{CatE}^{-/-}$mice fed an ND or HFD. The gross appearances of ND-fed wild-type and CatE $E^{-/}$mice were similar (data not shown). However, HFD-fed $\mathrm{CatE}^{-/-}$mice at 24 weeks exhibited markedly swollen and brownish-yellow livers compared to wild-type littermates (Fig. 1C). In addition, HFD-induced $\mathrm{CatE}^{-/-}$mice displayed a small amount of white adipose tissues (WATs), whereas HFD-induced wild-type mice displayed a large amount of WATs (Fig. 1C). These results suggest that HFD-induced $\mathrm{CatE}^{-/-}$mice exhibit abnormal lipid metabolism, which is characterized by hepatomegaly and reduced WAT development.

\subsection{Histopathological analysis of hepatomegaly in HFD-induced $\mathrm{CatE}^{-/-}$mice}

To further investigate the hepatomegaly observed in HFD-induced CatE ${ }^{-/-}$mice, histopathological analysis was performed (Fig. 2). In the liver of HFD-induced wild-type mice, hepatocytes around the centers of hepatic lobules were slightly swollen, and the small vesicles were prominent at the marginal regions of hepatic lobules (Fig. 2A). However, the liver of HFD-induced CatE $E^{-/}$mice contained many larger vacuoles in the cytoplasm (Fig. 2B and C). Moreover, the hepatic cell cord was irregularly shaped, and pseudoglandular transformation was pronounced in the hepatic lobule (Fig. 2B and C). These findings indicate that $\mathrm{CatE}^{-/-}$mice showed hepatomegaly associated with hepatic steatosis. 


\subsection{Hypercholesterolemia in HFD-induced $\mathrm{CatE}^{-/-}$mice}

To analyze the effects of the HFD on lipid metabolism in $\mathrm{CatE}^{-/-}$mice, we assessed serum lipid composition in wild-type and $\mathrm{CatE}^{-/-}$mice fed either an ND or HFD. The serum levels of total $\mathrm{CHO}$ and LDL in HFD-induced CatE ${ }^{-/}$mice were significantly higher than those in fat-induced wild-type mice (Fig. 3A and C). In contrast, serum levels of HDL in HFD-induced CatE ${ }^{-/-}$mice were markedly lower than those in wild-type mice (Fig. 3B). The serum levels of NEFA in wild-type and CatE ${ }^{-/-}$mice were equivalent (Fig. 3D). In ND-fed wild-type and CatE ${ }^{-/-}$mice, total CHO, LDL, HDL and NEFA levels were comparable (Fig. 3A-D).

To further examine the characteristics of HFD-induced Cat ${ }^{-/-}$mice, we investigated the inflammatory reactions and oxidative stress. We examined the serum levels of TBARS, which is an indicator for lipid peroxidation. For both ND or HFD-induced conditions, the serum TBARS levels in $\mathrm{CatE}^{-/}$mice were significantly higher than those of wild-type mice (Fig. 3E). To examine whether the HFD caused inflammation in $\mathrm{CatE}^{-/-}$mice, we measured SAA levels in wild-type and $\mathrm{CatE}^{-/-}$mice. The SAA levels in HFD-induced $\mathrm{CatE}^{-/-}$mice were markedly higher than those of the wild-type mice, although the levels were similar between wild-type and CatE ${ }^{-/}$ mice fed an ND (Fig. 3F). However, no notable differences were observed between the serum glucose or insulin levels of wild-type and $\mathrm{CatE}^{-/}$mice fed either an ND or HFD (Fig. 3G and H). These results indicate that HFD-induced $\mathrm{CatE}^{-/-}$mice display hypercholesterolemia associated with increased inflammatory reactions and oxidative stress, but not diabetes-like phenotypes.

\subsection{Impaired development of adipose tissues and reduced macrophage infiltration in HFD-induced CatE ${ }^{-/-}$mice}

To evaluate the impaired development of WATs in HFD-induced CatE ${ }^{-/-}$mice, we measured the weight of various adipose tissues in mice. As shown in Fig 4, $\mathrm{CatE}^{-/-}$mice displayed significantly reduced weights of epididymal WAT (Fig. 4A), perirenal WAT (Fig. 4B) 
and BAT (Fig. 4C). Histopathological analysis showed that adipocytes from epididymal WAT in $\mathrm{CatE}^{-/-}$mice were smaller than those of wild-type mice (Fig. 4D and E). Similarly, adipocytes from BAT in $\mathrm{CatE}^{-/-}$mice were significantly smaller than those in wild-type mice (Fig. 4F and H).

Finally, we explored the cellular roles of cathepsin E in adipose tissue development. Several lines of evidence indicate that cathepsin E is hardly detectable in pre-adipocyte cells [14]. Therefore, we hypothesized that macrophages, which contain higher levels of cathepsin E, may play an important role in the development of adipose tissues. When we performed immunohistochemical analysis using an antibody specific for the macrophage marker F4/80, immunoreactions for F4/80 in wild-type WAT were clearly detectable (Fig. 4H), whereas those in $\mathrm{CatE}^{-/-}$WAT were scarcely detectable (Fig. 4I). As shown in Fig. 4J, the number of F4/80-positive cells in $\mathrm{CatE}^{-/-}$WAT was significantly lower than that in wild-type WAT. These results indicate that impaired macrophage infiltration may be involved in the defective development of WAT.

\section{Discussion}

In this study, we have demonstrated that HFD-fed $\mathrm{CatE}^{-/-}$mice exhibit significantly reduced body weight gain and impaired development of WAT and BAT. This defective adipose tissue development was associated with hepatomegaly characterized by hepatic steatosis and hypercholesterolemia. Defective adipose tissue development in HFD-fed CatE ${ }^{-/}$mice is likely to be correlated to impaired macrophage infiltration.

Fat-induced $\mathrm{CatE}^{-/-}$mice exhibited impaired adipose tissue development. We speculate that the presence of abnormal adipose tissue is largely due to reduced macrophage infiltration in $\mathrm{CatE}^{-/-}$mice. It is known that the maturation process from pre-adipocyte to adipocyte is predominantly sustained by infiltration and interaction of macrophages and immune-related cells [15,16]. Moreover, the fact that cathepsin E is hardly detected in adipocytes but is highly 
expressed in macrophages further supports our notion. In fact, Cook et al. [14] reported that the mRNA level of cathepsin E was completely undetectable in 3T3-L1 cells, which is a useful cell line for adipocyte differentiation. In this study, the number of F4/80-positive cells in $\mathrm{CatE}^{-/-}$ WAT was significantly lower than that in wild-type WAT, indicating reduced macrophage infiltration in $\mathrm{CatE}^{-/-}$WAT. Therefore, it is likely that reduced adipose tissue development in $\mathrm{CatE}^{-/-}$mice is probably due to abnormalities in macrophage infiltration rather than in adipocytes. Consistent with this view, our previous study showed that $C a t E^{-/-}$macrophages have reduced pro-inflammatory cytokine production [9], impaired chemotaxis, and decreased cell adhesion [10]. Therefore, these findings also implicate $\mathrm{CatE}^{-/-}$macrophages in the decrease in adipose tissue development.

The phenotypes of fat-induced $\mathrm{CatE}^{-/-}$mice displaying defective adipose tissue development are reminiscent of those of mice deficient in cathepsin L, a ubiquitously distributed cysteine protease [17], or cathepsin $\mathrm{K}$, the osteoclast- and activated macrophage-specific cysteine protease [18] [19]. Fat-induced mice, deficient in either cathepsin L or cathepsin K, all display a lean phenotype and defective adipose tissue development. However, other characteristics of fat-induced $\mathrm{CatE}^{-/-}$mice such as hepatomegaly with hepatic steatosis and hypercholesterolemia, have not been reported in the fat-induced cathepsin L- or cathepsin K-deficient mice [20]. The precise mechanisms by which these three distinct types of cathepsin-deficient mice show partially similar phenotypes remain unknown. However, it is reasonable to speculate that the common adipose tissue defect in these mice is probably a primary event, while other phenotypes, including hepatomegaly with hepatic steatosis and hypercholesterolemia in fat-induced $\mathrm{CatE}^{-/-}$ mice, may be secondary events.

In conclusion, HFD-induced CatE ${ }^{-/}$mice exhibit impaired adipose tissue development that probably causes hepatomegaly associated with hepatic steatosis and hypercholesterolemia. This defective adipose tissue development may be caused in part by reduced macrophage infiltration. 


\section{Acknowledgements}

This work was supported in part by a Grant-in-Aid for Scientific Research from the Ministry of Education, Culture, Sports, Science, and Technology of Japan (T.T.). We would like to thank Prof. Keiichi I. Nakayama (Kyushu University, Department of Molecular and Cellular Biology, Medical Institute of Bioregulation) for generating the $\mathrm{CatE}^{-/-}$mice.

\section{Figure legends}

Figure 1. Changes in wild-type or $\mathrm{CatE}^{-/-}$mice fed an ND or HFD. Comparison of body weight gain in wild-type and $\mathrm{CatE}^{---}$mice. (A) Mice were continuously fed a normal diet (ND). (B) The ND was switched to a high-fat diet (HFD) when the mice were 8 weeks old. The arrow indicates the date of switching from an ND to an HFD. (C) Gross appearance after dissection of wild-type or $\mathrm{CatE}^{-/-}$mice under HFD conditions at 24 weeks.

Figure 2. Histopathological analysis of the liver from HFD-induced wild-type or CatE $^{-/-}$ mice. Hematoxylin-eosin staining of the liver from wild-type mice (A) and CatE-/- mice (B) (C) under HFD) conditions at 24 weeks. Bars: $100 \mu \mathrm{m}$ (A and B) or $50 \mu \mathrm{m}$.

Figure 3. Serum levels of various parameters for wild-type or $\mathbf{C a t E}^{-/-}$mice. Wild-type and CatE $^{-/-}$mice were fed an ND or HFD for 16 weeks and were fasted. The serum levels of cholesterol (CHO), high-density lipid (HDL), low-density lipid (LDL), nonesterified fatty acid (NEFA), thiobarbituric acid reactive substances (TBARS), serum amyloid A (SAA), glucose, and insulin were determined by the method described in Materials and Methods. (A) Total CHO; 
(B) HDL; (C) LDL; (D) NEFA. (E) TBARS; (F) SAA; (G) glucose; (H) insulin. The asterisk indicates the result of an unpaired Student's t-test comparing the values obtained between wild-type and $\mathrm{CatE}^{-/-}$mice, where $P<0.05$.

Figure 4. Analysis of adipose tissues from wild-type or CatE $^{-/-}$mice. Weights of epididymal white adipose tissue (WAT) (A), perirenal WAT (B), brown adipose tissue (BAT) (C) from wild-type or CatE ${ }^{-/-}$mice $(n=6)$ under high-fat diet (HFD) conditions at 24 weeks of age. Results are the mean \pm standard error of the mean $(\mathrm{SEM}) .{ }^{*} P<0.05$ versus the corresponding values obtained with the wild-type mice, obtained with the unpaired Student's $t$ test. Bars: $100 \mu \mathrm{m}$ (A and B). Histochemical analysis with hematoxylin-eosin staining of the epididymal WAT or BAT of wild-type mice (D and F respectively), and $\mathrm{CatE}^{-/-}$mice (E and G respectively). Bars: $100 \mu \mathrm{m}$ (D-G). Wild-type and CatE ${ }^{-/-}$mice were fed an HFD for 16 weeks and then fasted. Sections of epididymal WATs from wild-type mice (H) and CatE ${ }^{-/-}$mice (I) were stained with F4/80-specific antibody. (J) Number of F4/80-positive cells in the WAT of wild-type mice and $\mathrm{CatE}^{-/-}$mice. (slide number $=5$ ).

\section{References}

[1] K. Yamamoto, T. Kawakubo, A. Yasukochi, T. Tsukuba, Emerging roles of cathepsin E in host defense mechanisms, Biochim Biophys Acta 1824 (2012) 105-112.

[2] T. Tsukuba, K. Okamoto, Y. Yasuda, W. Morikawa, H. Nakanishi, K. Yamamoto, New functional aspects of cathepsin D and cathepsin E, Mol Cells 10 (2000) 601-611.

[3] T. Nishioku, K. Hashimoto, K. Yamashita, S.Y. Liou, Y. Kagamiishi, H. Maegawa, N. Katsube, C. Peters, K. von Figura, P. Saftig, N. Katunuma, K. Yamamoto, H. Nakanishi, Involvement of cathepsin $\mathrm{E}$ in exogenous antigen processing in primary cultured murine microglia, J Biol Chem 277 (2002) 4816-4822.

[4] H. Kakehashi, T. Nishioku, T. Tsukuba, T. Kadowaki, S. Nakamura, K. Yamamoto, Differential regulation of the nature and functions of dendritic cells and macrophages by cathepsin E, J Immunol 179 (2007) 5728-5737. 
[5] H. Sakai, T. Saku, Y. Kato, K. Yamamoto, Quantitation and immunohistochemical localization of cathepsins E and D in rat tissues and blood cells, Biochim Biophys Acta 991 (1989) 367-375.

[6] T. Kawakubo, A. Yasukochi, T. Tsukuba, T. Kadowaki, K. Yamamoto, Gene expression profiling of mammary glands of cathepsin E-deficient mice compared with wild-type littermates, Biochimie 90 (2008) 396-404.

[7] T. Tsukuba, K. Okamoto, Y. Okamoto, M. Yanagawa, K. Kohmura, Y. Yasuda, H. Uchi, T. Nakahara, M. Furue, K. Nakayama, T. Kadowaki, K. Yamamoto, K.I. Nakayama, Association of cathepsin E deficiency with development of atopic dermatitis, J Biochem 134 (2003) 893-902.

[8] M. Yanagawa, T. Tsukuba, T. Nishioku, Y. Okamoto, K. Okamoto, R. Takii, Y. Terada, K.I. Nakayama, T. Kadowaki, K. Yamamoto, Cathepsin E deficiency induces a novel form of lysosomal storage disorder showing the accumulation of lysosomal membrane sialoglycoproteins and the elevation of lysosomal pH in macrophages, J Biol Chem 282 (2007) 1851-1862.

[9] T. Tsukuba, S. Yamamoto, M. Yanagawa, K. Okamoto, Y. Okamoto, K.I. Nakayama, T. Kadowaki, K. Yamamoto, Cathepsin E-deficient mice show increased susceptibility to bacterial infection associated with the decreased expression of multiple cell surface Toll-like receptors, J Biochem 140 (2006) 57-66.

[10] T. Tsukuba, M. Yanagawa, K. Okamoto, Y. Okamoto, Y. Yasuda, K.I. Nakayama, T. Kadowaki, K. Yamamoto, Impaired chemotaxis and cell adhesion due to decrease in several cell-surface receptors in cathepsin E-deficient macrophages, J Biochem 145 (2009) 565-573.

[11] H. Du, M. Heur, M. Duanmu, G.A. Grabowski, D.Y. Hui, D.P. Witte, J. Mishra, Lysosomal acid lipase-deficient mice: depletion of white and brown fat, severe hepatosplenomegaly, and shortened life span, J Lipid Res 42 (2001) 489-500.

[12] J.C. Woloszynek, T. Coleman, C.F. Semenkovich, M.S. Sands, Lysosomal dysfunction results in altered energy balance, J Biol Chem 282 (2007) 35765-35771.

[13] M.A. Kido, T. Yamaza, T. Goto, T. Tanaka, Immunocytochemical localization of substance P neurokinin-1 receptors in rat gingival tissue, Cell Tissue Res 297 (1999) 213-222.

[14] M. Cook, R.C. Caswell, R.J. Richards, J. Kay, P.J. Tatnell, Regulation of human and mouse procathepsin E gene expression, Eur J Biochem 268 (2001) 2658-2668.

[15] V. Bourlier, A. Bouloumie, Role of macrophage tissue infiltration in obesity and insulin resistance, Diabetes Metab 35 (2009) 251-260. 
[16] A. Sorisky, A.S. Molgat, A. Gagnon, Macrophage-induced adipose tissue dysfunction and the preadipocyte: should I stay (and differentiate) or should I go?, Adv Nutr 4 (2013) 67-75.

[17] M. Yang, Y. Zhang, J. Pan, J. Sun, J. Liu, P. Libby, G.K. Sukhova, A. Doria, N. Katunuma, O.D. Peroni, M. Guerre-Millo, B.B. Kahn, K. Clement, G.P. Shi, Cathepsin L activity controls adipogenesis and glucose tolerance, Nat Cell Biol 9 (2007) 970-977.

[18] M. Yang, J. Sun, T. Zhang, J. Liu, J. Zhang, M.A. Shi, F. Darakhshan, M. Guerre-Millo, K. Clement, B.D. Gelb, G. Dolgnov, G.P. Shi, Deficiency and inhibition of cathepsin K reduce body weight gain and increase glucose metabolism in mice, Arterioscler Thromb Vasc Biol 28 (2008) 2202-2208.

[19] M. Funicello, M. Novelli, M. Ragni, T. Vottari, C. Cocuzza, J. Soriano-Lopez, C. Chiellini, F. Boschi, P. Marzola, P. Masiello, P. Saftig, F. Santini, R. St-Jacques, S. Desmarais, N. Morin, J. Mancini, M.D. Percival, A. Pinchera, M. Maffei, Cathepsin K null mice show reduced adiposity during the rapid accumulation of fat stores, PLoS One 2 (2007) e683.

[20] J.C. Lafarge, N. Naour, K. Clement, M. Guerre-Millo, Cathepsins and cystatin C in atherosclerosis and obesity, Biochimie 92 (2010) 1580-1586. 
A B

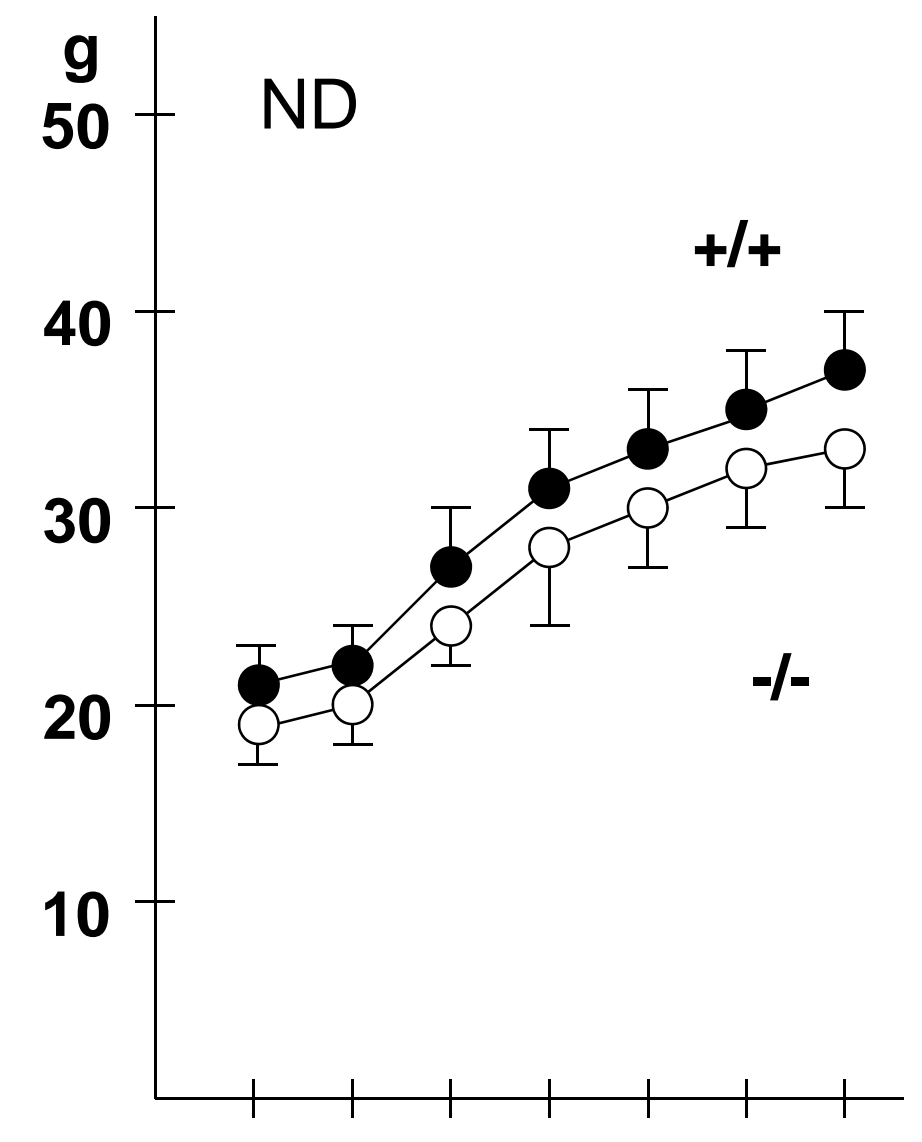

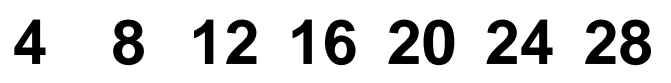
weeks
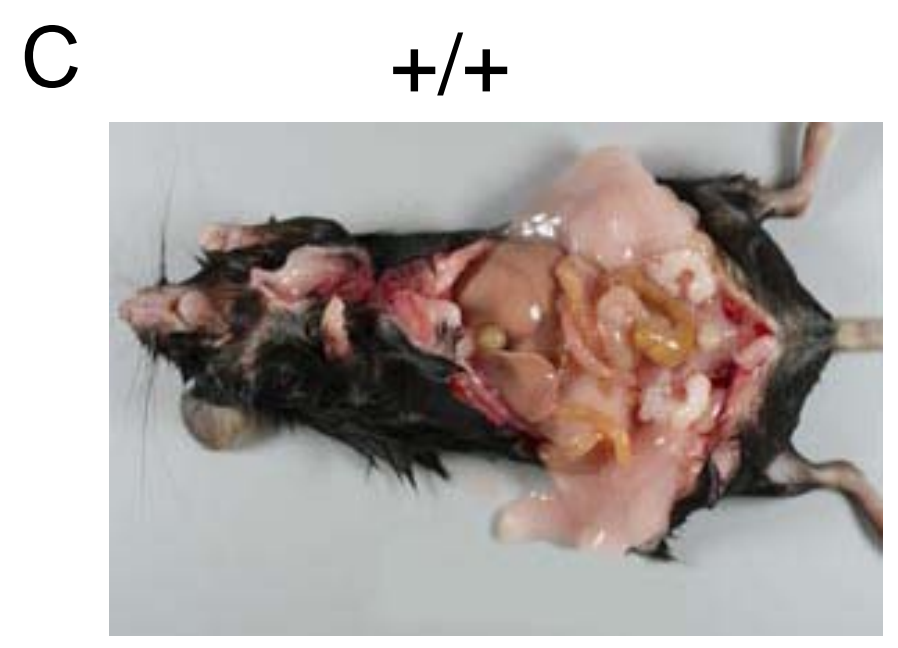

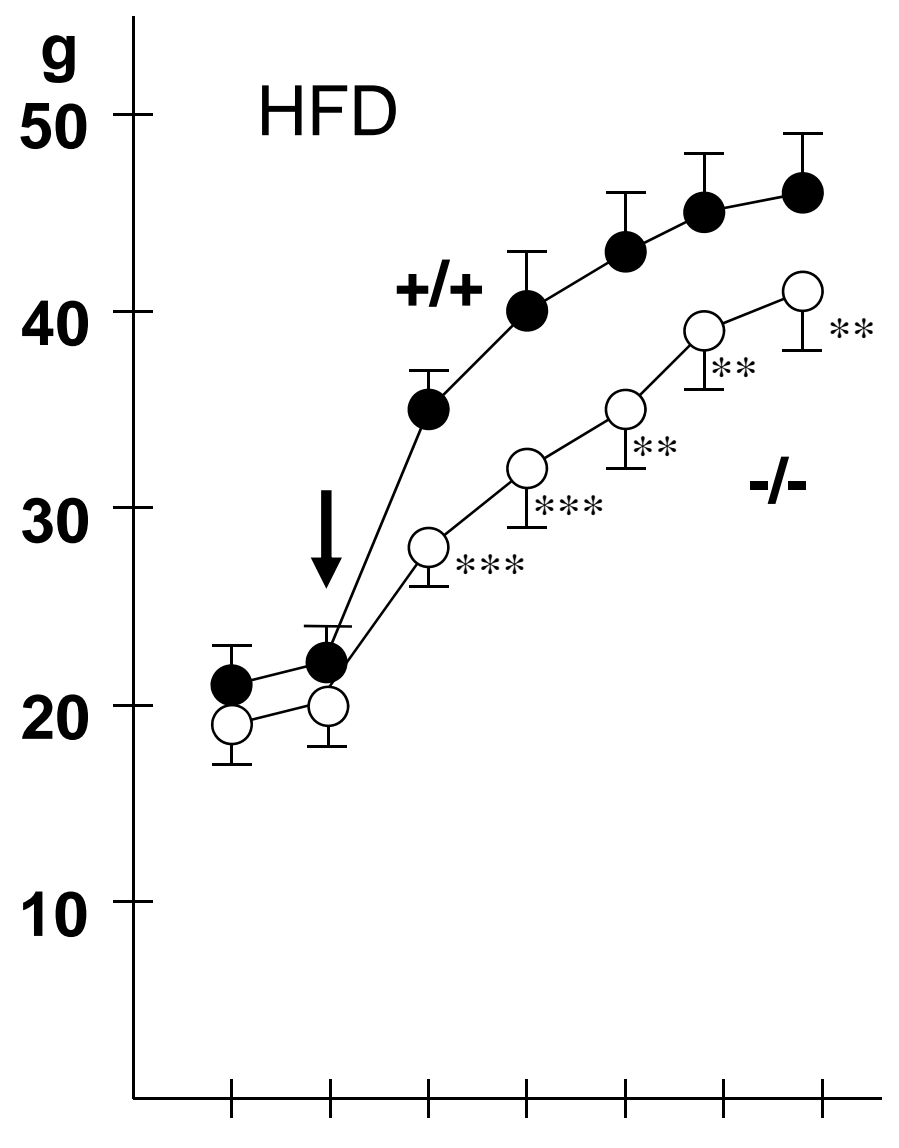

$\begin{array}{lllllll}4 & 8 & 12 & 16 & 20 & 24 & 28\end{array}$ weeks

$-1-$

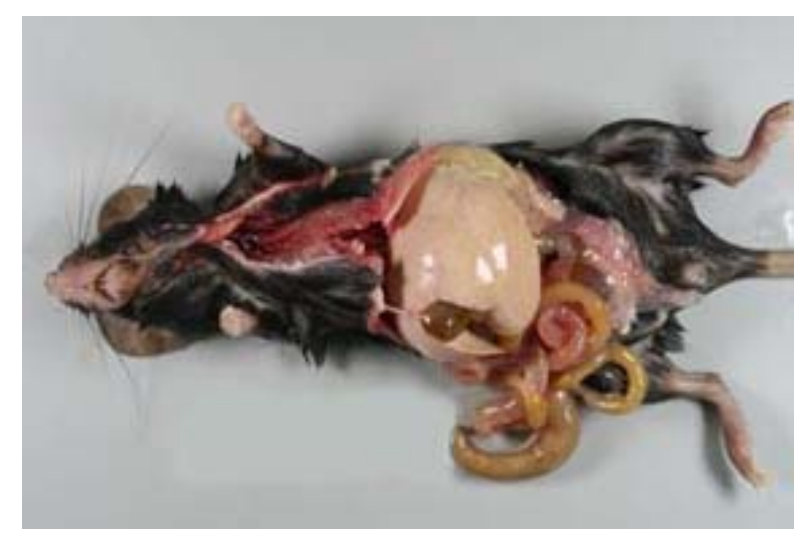

Figure 1 
A

$+/+$

B $\quad-/-$
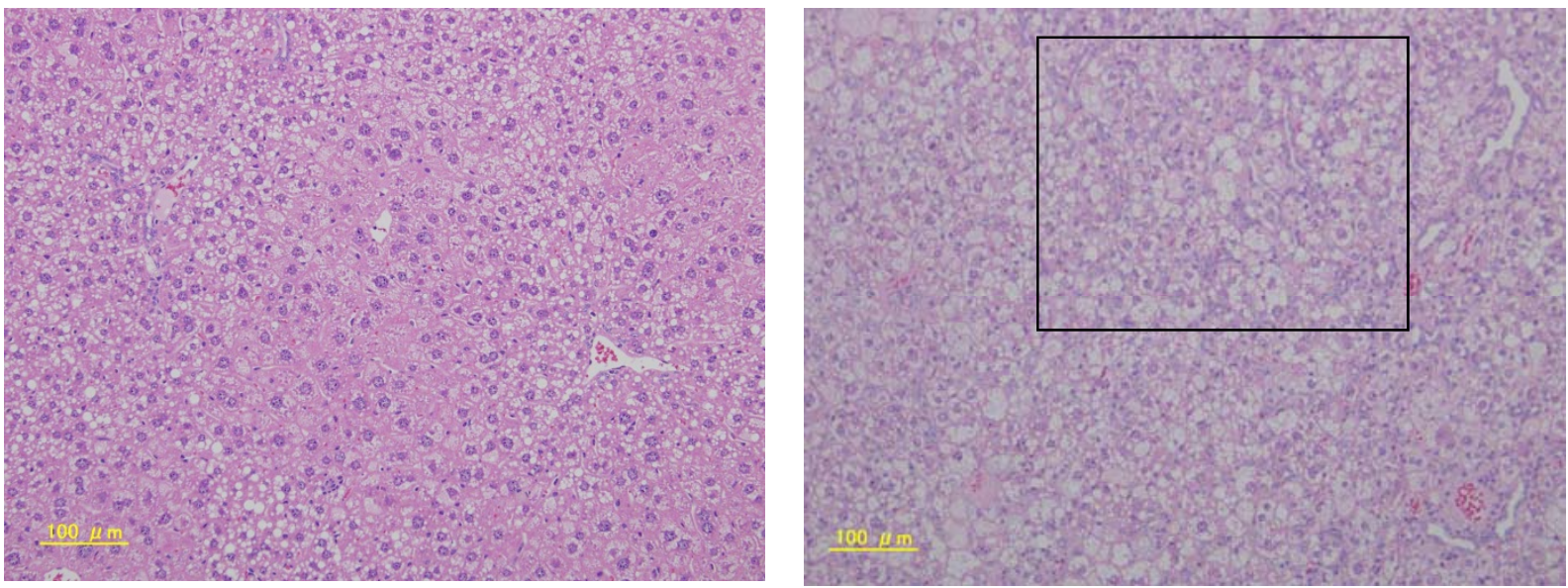

C

$-/-$

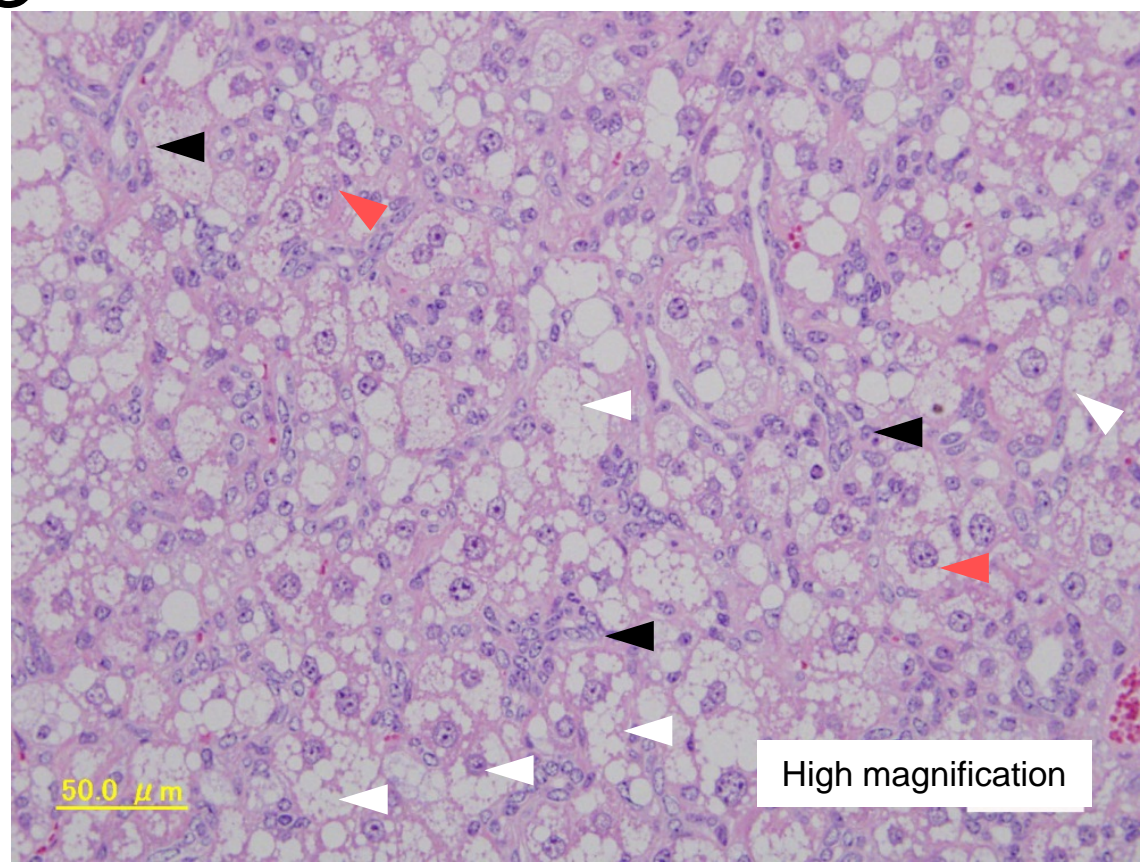

Figure 2 

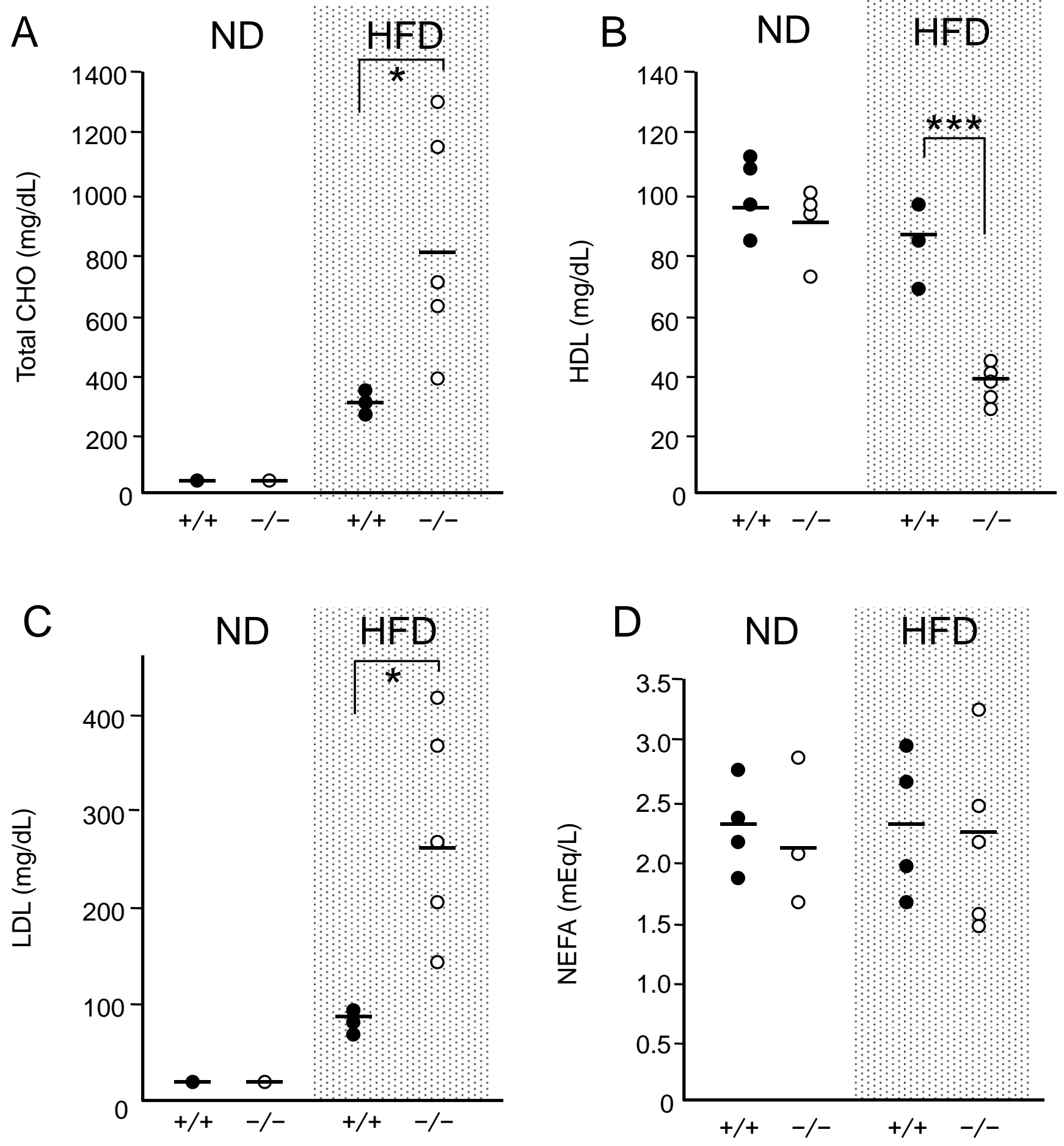

Figure 3 (to be continued) 

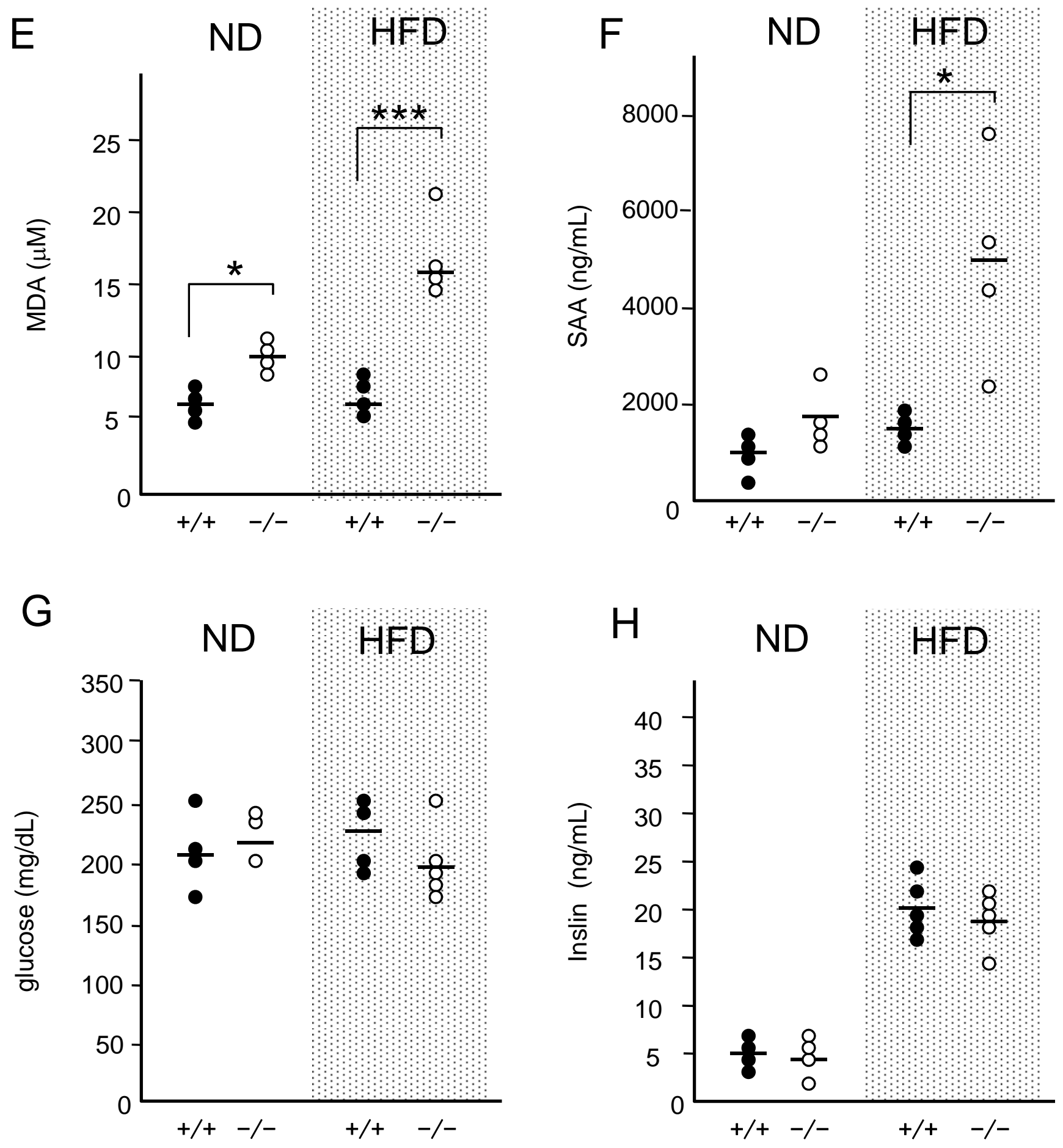

Figure 3 
$A_{\text {Epididymal WAT }} B$ Perirenal WAT $\quad C$ BAT
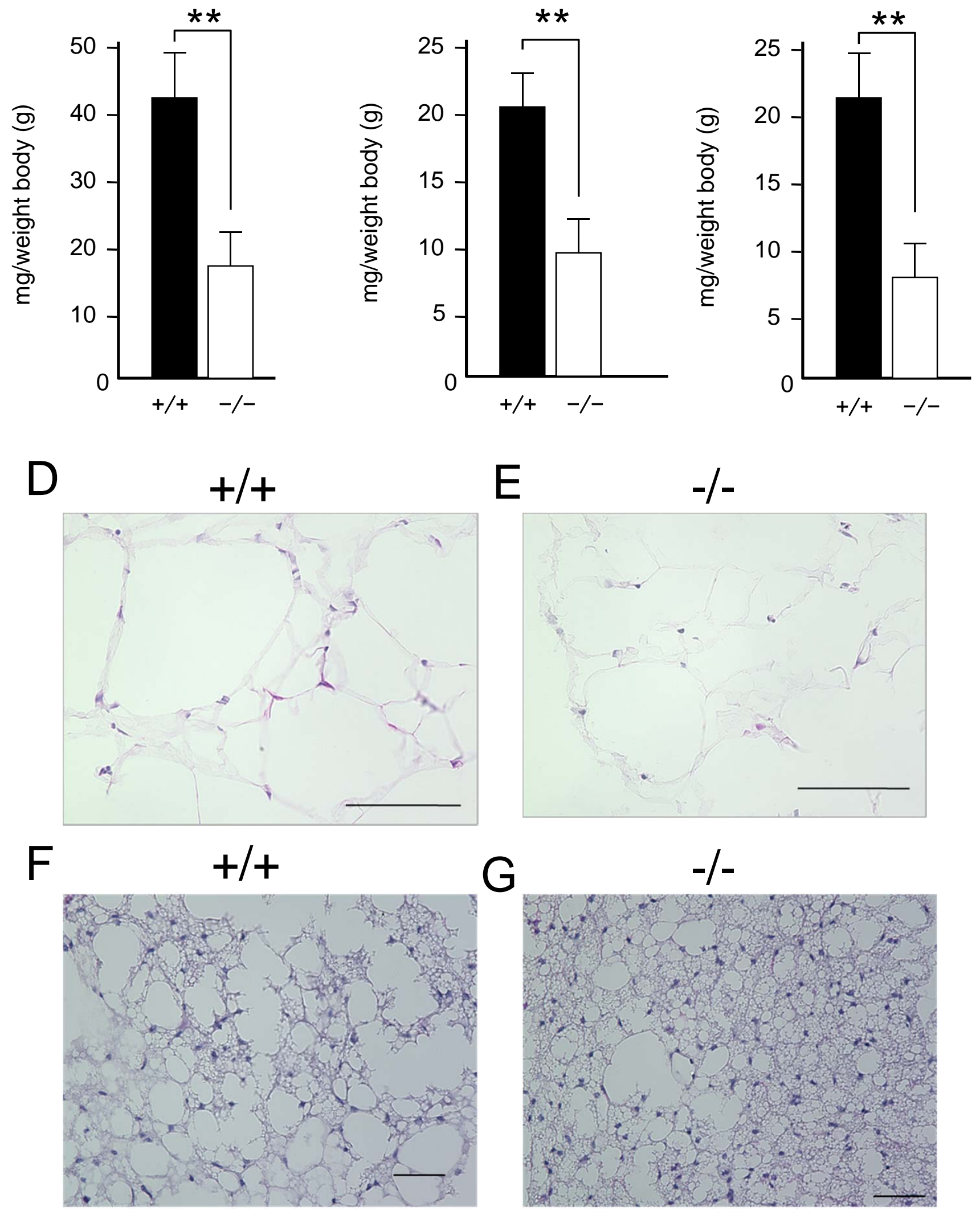

Figure 4 (to be continued) 

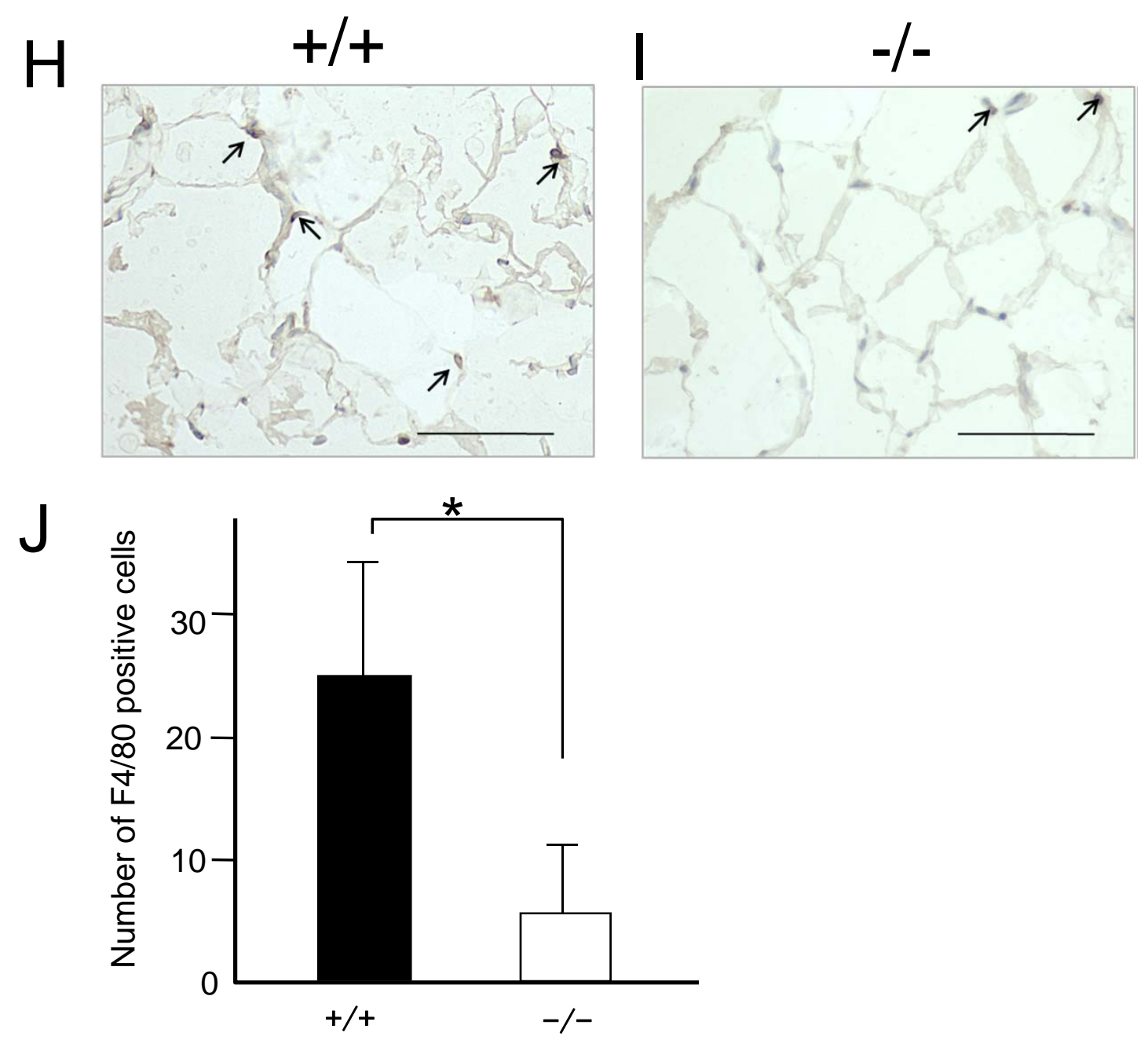

Figure 4 